\title{
Adsorption of Natural Lung Surfactant and Phospholipid Extracts Related to Tubular Myelin Formation
}

\author{
R. H. NOTTER, D. P. PENNEY, J. N. FINKELSTEIN, and D. L. SHAPIRO \\ Departments of Pediatrics (Division of Neonatology) [R.H.N., J.N.F., D.L.S.], Anatomy [D.P.P.], and Cancer \\ Center [D.P.P.], University of Rochester School of Medicine, Rochester, New York 14642
}

\begin{abstract}
The microstructure of aqueous dispersions of pulmonary surfactant lipids extracted from bovine lung lavage was investigated by electron microscopic analysis. Following organic solvent extraction (chloroform/methanol) from natural lung surfactant, the mixed lipids (CLL), with $1 \%$ residual protein, were dispersed in water by two techniques, probe sonication at $4^{\circ} \mathrm{C}$ and mechanical vortexing. Surface pressure-time adsorption isotherms were defined for the CLL dispersions, followed by staining with tannic acid, uranyl acetate, and lead citrate for electron microscopy of microstructure. CLL extract dispersions adsorbed in seconds to surface pressures near $45 \mathrm{dynes} / \mathrm{cm}$ (surface tension 25 dynes/cm) at low concentrations $\leq 0.25$ $\mathrm{mg} / \mathrm{ml}$ after dispersion by sonication of $4^{\circ} \mathrm{C}$ and by mechanical vortexing. Ultrastructurally, the CLL dispersions were somewhat heterogeneous, but large thin-walled phospholipid vesicles, both intact and fragmented, predominated. No tubular myelin was formed. By contrast, natural lung surfactant (LS) from bronchoalveolar lavage had characteristic regions of tubular myelin when it adsorbed well at low concentrations ( $\leq \mathbf{0 . 2 5} \mathrm{mg}$ phospholipid $/ \mathrm{ml}$ ). When divalent cations were removed from solution with $5 \mathrm{mM}$ EDTA, this distinctive microstructure was not present and natural LS adsorbed less rapidly; higher concentrations of $0.63 \mathrm{mg}$ phospholipid/ml were necessary for maximal adsorption of natural LS without tubular myelin. These results suggest that while tubular myelin is associated with optimal adsorption for natural $\mathbf{L S}$, it is not a required configuration for rapid adsorption facility at low phospholipid concentrations in general. Specifically, for dispersions of surfactant extracts, other microstructures allow adsorption facility equivalent to that of natural LS with tubular myelin. (Pediatr Res 20:97-101, 1986)
\end{abstract}

CLL, calf lung lipids

LS, natural lung surfactant

$\mathrm{V}$, dispersion by vortexing at room temperature

$\mathrm{S}_{\mathrm{i}}$, dispersion by sonication at $4^{\circ} \mathrm{C}$

$\pi$-t, surface pressure-time

RDS, respiratory distress syndrome

\section{Abbreviations}

The behavior of phospholipid dispersions in an aqueous bulk phase is directly relevant to the RDS of premature neonates which is due to a deficiency of pulmonary surfactant $(1,2)$. In the mature lung, pulmonary surfactant undergoes a series of structural changes as it is released from the lamellar bodies of type II pneumocytes, enters the aqueous alveolar hypophase, and adsorbs to the air-water interface. A number of studies with a variety of models, including alveolar type II cells in culture, have demonstrated the distinctive LS microstructure known as tubular myelin when secreted LS is present in an aqueous bulk phase (e.g. References 3-6). This LS microstructure appears to be associated closely with adsorption facility, and surfactant associated apoproteins are considered to play an important role in tubular myelin formation in natural LS $(5,6)$.

The administration of exogenous surfactants to the lungs is a promising approach in neonatal RDS therapy (e.g. References 2, 7-11) and may also have applications in other lung disorders such as the adult respiratory distress syndrome (e.g. References $8,12)$. For in vivo effectiveness, replacement surfactants must have a solution structure which promotes adsorption to the airwater interface after pulmonary delivery. In general, exogenous lung surfactants contain a high percentage of phospholipids, and phospholipid adsorption can be a function of the technique used for dispersion in the aqueous bulk phase $(8,13,14)$. This behavior is related to the ability of phospholipids to form different microstructural states in water, including smectic mesophases and liposomal $^{1}$ aggregates. However, rigorous physicochemical descriptions of the relations between dispersion microstructure and phospholipid molecule adsorption (or phospholipid-protein adsorption) are not now available.

The present study represents an empirical approach to investigate relations between dispersion microstructure and adsorption facility for an organic solvent extract of natural LS. This material, extracted CLL containing approximately $1 \%$ residual protein, reversed surfactant-deficient pressure-volume mechanics in excised lungs (19), improved lung function in premature lambs of 18 and 19 wk gestation $(8,20,21)$, and was an effective treatment for human infants with $\operatorname{RDS}(9,10)$. Beneficial effects on surfacant-deficient lungs have also been found for closely related LS extracts by other investigators (e.g. References 7, 11, 22 ). Since the $1 \%$ residual protein in the CLL extract was necessary for optimal adsorption (14), we asked whether rapidly adsorbing CLL dispersions would exhibit the tubular myelin-like microstructure characteristic of natural LS.

Received May 13, 1985; accepted August 21, 1985.

Address for correspondence R. H. Notter, Department of Pediatrics-Box 777, Division of Neonatology, University of Rochester, Rochester, NY 14642.

This study was supported by NIH grants HL-25170, CA-11109, CA-27791, and HL-00945 (RCDA to RHN).
'The terminology "liposome" as used here describes the closed, vesicle-like phospholipid forms first reported by Bangham and Horne (15), which have been the object of many previous electron microscopic studies (see References 16-18 for reviews). 


\section{MATERIALS AND METHODS}

Calf lung lavage surfactant and CLL extract. Surfactant was obtained by lavage from the lungs of freshly sacrificed calves (Conti Packing Co., Henrietta, NY), as described previously (8, $13,14)$. Lungs, with trachea and bronchi intact, were lavaged with 3 liters per lung of $\operatorname{cool}\left(20^{\circ} \mathrm{C}\right) 0.15 \mathrm{M} \mathrm{NaCl}$, given in three divided doses. The lavage fluid was centrifuged at $300 \times g$ for 10 min to remove cellular debris. The supernatant was drawn off and centrifuged at $12,000 \times g$ for $30 \mathrm{~min}$ at $4^{\circ} \mathrm{C}$ to pellet the surfactant material. This material, not subjected to further treatment in terms of extraction or in terms of mechanical suspension (sonication or vortexing), is referred to here as whole LS. It is comparable in adsorption behavior to the surface active fractions of animal lung lavage obtained by more elaborate centrifugation procedures $(5,6)$.

The LS obtained by lavage was divided into two fractions. One fraction was resuspended in $0.15 \mathrm{M} \mathrm{NaCl}$ and was used in all natural LS adsorption and microstructure experiments. The second LS fraction was treated with chloroform-methanol by the technique of Bligh and Dyer (23) to extract the lipids. These extracted lung lipids, designated CLL, are representative of the lipid extracts used successfully in previous exogenous surfactant replacement studies in excised rat lungs (19), in premature rabbit fetuses (22), in premature lambs in vivo $(20,21)$, and in infants with RDS (9-11).

The CLL extract from lavaged LS contained 91 weight $\%$ phospholipid, analyzed with the microphosphorus techinique of Ames (24). Thin-layer chromatography analysis with the solvent system of Touchstone et al. (25) showed the phospholipid to contain $83 \%$ phosphatidylcholine, $3.2 \%$ phosphatidylglycerol, $2.7 \%$ phosphatidylethanolanolamine, $5.8 \%$ phosphatidylinositol (+phosphatidylserine), $2.7 \%$ sphingomyelin, and $2.6 \%$ other. The phosphatidylcholine fraction of CLL was analyzed by gas chromagraphy to have a fatty acid distribution of $\mathrm{C} 14: 0,6.2 \%$; $\mathrm{C} 16: 0,68.5 \%, \mathrm{C} 16: 1,10.2 \%$; $\mathrm{C} 18: 0,3.2 \%$; $18: 1,7.5 \%$; other, 4.6\%. The CLL contained 5 weight \% cholesterol and cholesterol esters, and had a protein content of 1 weight $\%$ by a modification of the method of Lowry et al. (26) which allowed determination of protein in the presence of lipid. By comparison, the protein content of natural LS was about an order of magnitude higher at $10 \%$ by weight.

Adsorption $\pi-t^{2}$ measurements. Adsorption $\pi$ - $\mathrm{t}$ isotherms were measured as described in detail by Notter et al. $(13,14)$. Briefly, experiments were done in a Teflon dish with a $70 \mathrm{ml}$ subphase. For CLL experiments the subphase was $0.15 \mathrm{M} \mathrm{NaCl}$, as used most typically in exogenous surfactant replacement studies $(9$, $10,20,21)$. For natural LS, the subphase was sodium and potassium phosphate buffered saline, $\mathrm{pH} 7.4$, which was supplemented with divalent metal ions or EDTA as noted. In all adsorption experiments, the subphase was stirred continuously by a Teflon-coated magnetic stirring bar to minimize diffusion resistance. At time zero, a volume of $10 \mathrm{ml}$ of $0.15 \mathrm{M} \mathrm{NaCl}$ or phosphate buffered saline containing a specified amount of CLL or LS (based on lipid phosphorus) was added as a bolus to the $70 \mathrm{ml}$ subphase. Surface pressure was then monitored continuously by measurement of the force on a sandblasted platinum slide dipped into the surface (temperature $35 \pm 2^{\circ} \mathrm{C}$ ).

Dispersions of CLL extract for adsorption and electron microscopic analyses were formed by the two techniques most widely used in surfactant replacement studies with extracted lipid preparations $(8-11,19-22)$, i.e. probe sonication and vortexing. Sonication was continuous in an ice bath for $1 \min (25 \mathrm{~W}$, microtip, Heat Systems-Ultrasonic, Inc., Plainview, NY), repeated up to three times as necessary to disperse visible particles, designated $\left(\mathrm{S}_{\mathrm{i}}\right)$. Mechanical vortexing (Four Tube Vortex Mixer, Scientific Industries, Inc., Bohemia, NY) was for 1 h at ambient conditions $\left(22^{\circ} \mathrm{C}\right)$ without temperature control of the dispersion,

\footnotetext{
${ }^{2}$ Surface pressure $\pi$ is defined as the amount by which the surfactant lowers the surface tension of the pure subphase at the experimental temperature.
}

designated (V). For experiments correlating natural LS adsorption and microstructure, no artificial dispersion methods were used. For the case of optimal LS adsorption where a tubular myelin microstructure was expected, $1.4 \mathrm{mM} \mathrm{CaCl}_{2}$ plus 1.0 $\mathrm{mM} \mathrm{MgCl}$ (denoted $\mathrm{M}^{++}$) was added to the $10 \mathrm{ml}$ surfactant suspension and to the $70 \mathrm{ml}$ phosphate buffered saline adsorption subphase. The contrasting case of less rapid LS adsorption was with $5 \mathrm{mM}$ EDTA in the suspension medium and adsorption subphase, with no divalent metal ions added.

The general procedure for all adsorption and microstructure experiments was that surfactants were dispersed (CLL) or resuspended (natural LS) in $6 \mathrm{ml}$ of appropriate solution, at a concentration of $10 \mathrm{mg} / \mathrm{ml}$. Aliquots were then taken and diluted for use in replicate adsorption experiments. Duplicate $1.5-\mathrm{ml} \mathrm{sam-}$ ples were also taken and analyzed by electron microscopy as described below. This procedure meant that adsorption experiments were done on material from the same dispersion which was being examined microscopically for ultrastructure.

Electron microscopy methods. To each of the $1.5 \mathrm{ml}$ volumes of dispersed phospholipids was added an equal volume of $2 \%$ tannic acid in $0.1 \mathrm{M}$ sodium cacodylate buffer, $\mathrm{pH}$ 7.2. After 30 min fixation, the mixtures were washed several times in buffer and pelleted at $4000 \mathrm{rpm}$ for $20 \mathrm{~min}$ after each wash to remove the excess tannic acid, the supernatant being discarded (27). Each pellet was then fixed for $1 \mathrm{~h}$ in $1 \% \mathrm{OsO}_{4}$ in $0.1 \mathrm{M}$ sodium cacodylate buffer, $\mathrm{pH} 7.2$, and dehydrated in increasing concentrations of ethanol (27). Embedment was in Spurr (Polysciences, Warrington, PA) low viscosity resin. Thick $(0.5-1.0 \mu \mathrm{m})$ sections of epoxy-embedded phospholipids were stained with methylene blue-azure II for light microscopy and orientation. Pelleted material from each sample appeared homogeneous when viewed by light microscopy, and representative areas were selected for ultramicrometry. Thin $(40-80 \mathrm{~nm})$ sections were stained with uranyl acetate and lead citrate for observation and photography in a Zeiss $10 \mathrm{~A}$ electron microscope.

\section{RESULTS}

A representative $\pi$-t adsorption isotherm for $\operatorname{CLL}\left(\mathrm{S}_{\mathrm{i}}\right)$ and CLL(V) dispersions is shown in Figure $1 A$ for a bulk concentration of $0.25 \mathrm{mg} \mathrm{CLL} / \mathrm{ml}$. Both types of CLL dispersion adsorb extremely rapidly to high surface pressure at this low concentration. Further increases in concentration have little effect on CLL(V or $\mathrm{S}_{\mathrm{i}}$ ) adsorption because these dispersions are already adsorbing in a few seconds to the maximum value possible, i.e. to the highest equilibrium spreading pressure of the mixed film components, which for phospholipids is $45-50$ dynes $/ \mathrm{cm}(e . g$. Reference 8).

Figure $1 B$ shows $(\pi-\mathrm{t})$ isotherms for natural LS which demonstrate rapid adsorption in the presence of $\mathrm{Ca}^{++}$ions, and poorer adsorption at equivalent concentration if the chelating agent EDTA is present and divalent metal ions are not added. As for CLL extract adsorption, isotherm 1 in Figure $1 B$ is characteristic of maximal adsorption for natural LS, and does not change as concentration is increased above $0.25 \mathrm{mg} \mathrm{LS} / \mathrm{ml}$ (based on LS phospholipid). However, the inhibition of LS adsorption by EDTA is concentration dependent in Figure $1 B$. Although adsorption is retarded in the presence of $5 \mathrm{mM}$ EDTA at low concentration $(0.25 \mathrm{mg} \mathrm{LS} / \mathrm{ml})$, this effect is eliminated at higher concentrations of LS phospholipid $(0.63 \mathrm{mg} / \mathrm{ml})$.

Microstructural details of the dispersions characterized for adsorption in Figure 1 are shown in the electron micrographs of Figures 2 and 3 . Figure $2 A$ gives the ultrastructure of natural LS suspensions for the rapidly adsorbing case shown in Figure $1 B$ (curve 1 , in the presence of $1.4 \mathrm{mM} \mathrm{Ca}^{++}+1.0 \mathrm{mM} \mathrm{Mg}^{++}$). Figure $2 A$ is at a magnification of 17,500 and shows a number of areas of tubular myelin in the LS suspension. In contrast to these results, Figure $2 B$ shows the ultrastructure of LS for the case of less rapid adsorption in the presence of $5 \mathrm{mM}$ EDTA. The major microstructural difference is that no tubular myelin 

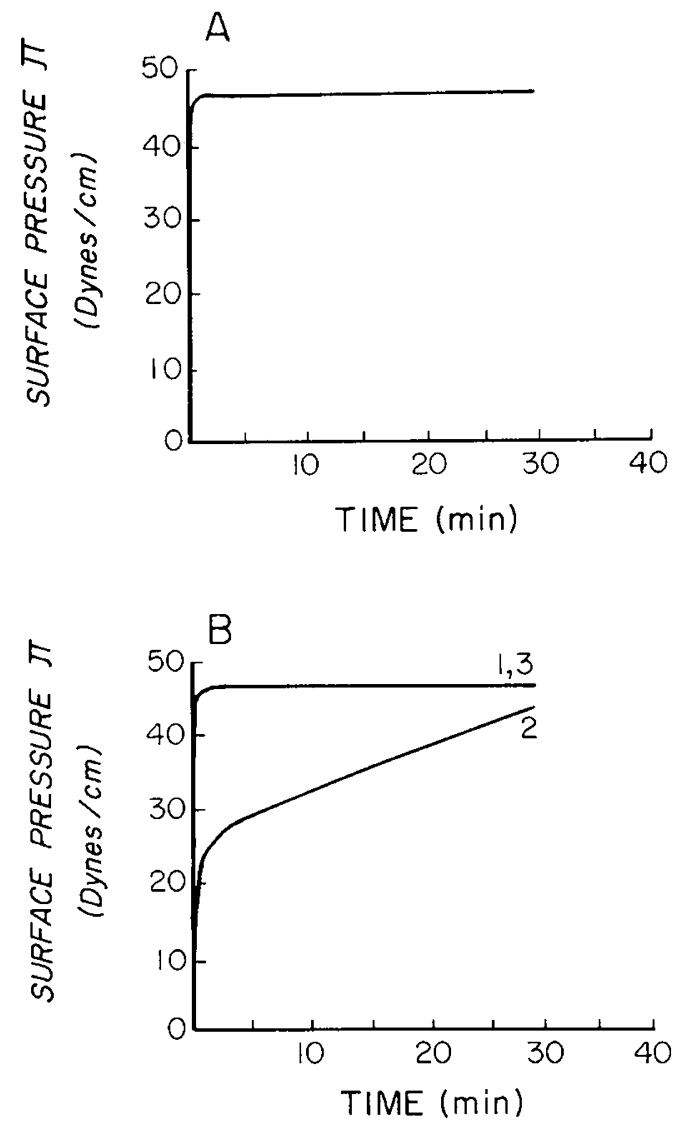

Fig. 1. Surface pressure-time adsorption isotherms for LS and aqueous dispersions of extracted surfactant phospholipids (CLL) at $35^{\circ}$ C. A, CLL dispersed $\left(\mathrm{S}_{\mathrm{i}}\right.$ or $\left.\mathrm{V}\right)$ in $0.15 \mathrm{M} \mathrm{NaCl}$ to a final subphase concentration of $0.25 \mathrm{mg} \mathrm{CLL} / \mathrm{ml}$. $B$, Natural LS resuspended in phosphate buffered saline: (1) concentration $0.25 \mathrm{mg}$ LS phospholipid/ml, with $1.4 \mathrm{mM} \mathrm{CaCl}_{2}+1.0 \mathrm{mM} \mathrm{MgCl}_{2}$; (2) concentration $0.25 \mathrm{mg} \mathrm{LS/}$ $\mathrm{ml}$, with $5 \mathrm{mM}$ EDTA; (3) concentration $0.63 \mathrm{mg} \mathrm{LS} / \mathrm{ml}$, with $5 \mathrm{mM}$ EDTA. See "Materials and methods" for specific subphase and dispersion conditions, and text for details.

is observed in LS suspensions to which EDTA has been added. Instead the microstructure is generally characterized by a heterogenous population of liposomes, a number of which are multilamellar.

The microstructure of sonicated and vortexed dispersions of solvent-extracted LS lipids, i.e. CLL( $\left.\mathrm{S}_{\mathrm{i}}\right)$ and CLL(V), are shown in Figure 3. The most significant feature of the CLL ultrastructural results is that tubular myelin is not present for either $S_{i}$ or $\mathrm{V}$ dispersions despite their excellent adsorption facility. ${ }^{3}$ For the $\operatorname{CLL}\left(\mathrm{S}_{\mathrm{i}}\right)$ dispersion in Figure $3 A$, there are clear subpopulations of unfolding liposomes, and open appearing networks, as well as large cistern-like structures. It is likely that the open-appearing networks in the electron micrograph thin sections in Figure $3 \mathrm{~A}$ represent both intact and fragmented regions from the walls of large, thin-walled liposomes. However, this interpretation is not definitive without detailed analysis of serial sections, which was not done in our present study. The term open-appearing is used here phenomenologically to refer to ribbon-like structures which are not closed liposomes in the plane of the section. The microstructure of the CLL(V) dispersion in Figure $3 B$ is somewhat less

\footnotetext{
${ }^{3}$ The CLL micrographs in Figure 3 are at a magnification of 17,500 , but higher power observations up to magnification of 100,000 failed to demonstrate any tubular-myelin like structures (data not shown explicitly). Note that this finding does not rule out the possibility that some dispersion condition or subphase ionic environment not studied here could lead to tubular myelin formation for a CLL extract dispersion. The point for these experiments is that CLL dispersions can be formulated to give rapid adsorption equivalent to natural LS without the necessity for this distinctive microstructure.
}
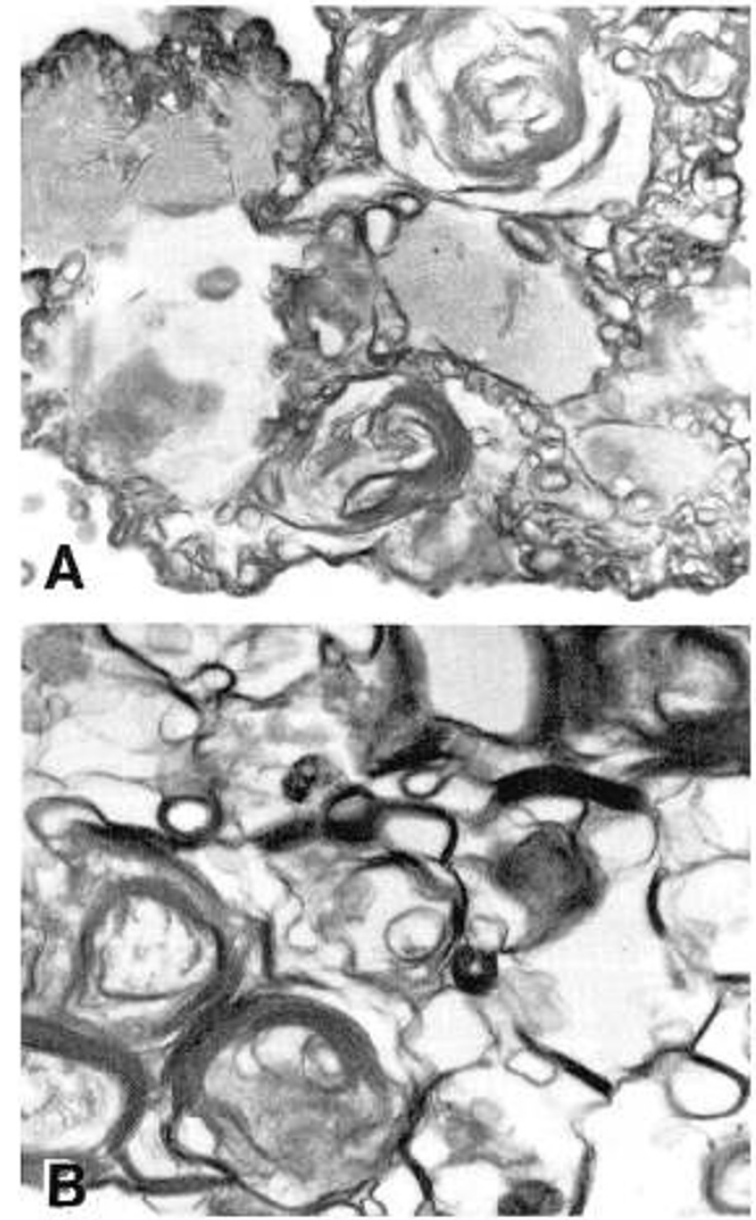

Fig. 2. Electron micrographs of natural LS suspensions for conditions of Figure $1 \mathrm{~B}$. A, rapid adsorption case corresponding to Figure $1 B$, curve $1(\times 17,500)$. $B$, less rapid adsorption case corresponding to Figure $1 B$, curves 2 and $3(\times 17,500)$. See "Materials and Methods" for dispersion fixation for electron microscopy.

heterogeneous than for the sonicated dispersion in Figure $3 \mathrm{~A}$, and contains a significant population of thin-walled, relatively large liposomes.

\section{DISCUSSION}

These experiments investigated relations between the microstructure and adsorption facility of dispersions of lung surfactant phospholipid extracts and of natural LS itself. The results in Figures 1 and 3 show that mixed lung lipids (CLL) with $1 \%$ protein, extracted in organic solvents from lavaged natural LS, adsorb well after aqueous dispersion without a tubular myelin microstructure. Both sonicated and vortexed CLL dispersions were studied. Although the ultrastructure of CLL $\left(\mathrm{S}_{\mathrm{i}}\right)$ dispersions was heterogenous, it included thin-walled liposomes plus regions of open-appearing, ribbon-like networks, consistent with sections of large thin-walled liposomes and unfolded liposome fragments. CLL(V) dispersion microstructure also showed a general characteristic of large thin-walled liposomes. The microstructural features found for rapidly adsorbing CLL dispersions are consistent with results reported by Obladen et al. (28), who studied the microstructure of a binary mixture of synthetic phospholipids, 9:1 DPPC:dipalmitoyl phosphatidylglycerol. They found that rapid adsorption correlated with the presence of large unilamellar liposomes in dispersions of this synthetic phospholipid mixture. We have also performed similar experiments with $7: 3$ DPPC:egg-PG mixtures dispersed by several techniques, and found microstructural features very close in appearance to those 
in Figure 3 to be associated with rapid adsorption (Nottor RH, Penney DP, Finkelstein JN, Shapiro DL, unpublished data).

Previous adsorption studies have suggested that the $1 \%$ protein in CLL is of probable importance for maximizing adsorption facility relative to synthetic phospholipids alone (14). The microstructural results in Figure 3 show that the protein in CLL does not induce tubular myelin formation in order to accomplish this enhanced adsorption at low phospholipid concentration, as is the case for natural LS in Figure 2. With regard to general correlations between adsorption facility and dispersion microstructure, our results suggest that the presence of phospholipids in an open microstructure is a prerequisite for optimal adsorption to the air-water interface. For the case of natural LS, this involves changes from a highly ordered structure within the lamellar body to the unfolded network of tubular myelin in the alveolar hypophase prior to entry at the air-water interface. Other LS microstructures also allow rapid adsorption to the maximum spreading pressure, but larger subphase concentrations are required compared to the case when tubular myelin is present (Figs. $1 B$ and $2 B$ ). The surfactant apoprotein (with $\mathrm{Ca}^{++}$) apparently plays a major role in the molecular interactions leading to the preferred tubular myelin form for natural $\operatorname{LS}(5,6)$. However, for surfactant extracts such as CLL, microstructures different from tubular myelin, but still conducive to rapid adsorption at equivalent low concentrations, ${ }^{4}$ can be achieved by mechanical dispersion methods.

A consistent interpretation is that rapidly adsorbing phospholipid dispersions must achieve a microstructure where structural defects exist, allowing for fracture and unfolding of liposomes. It is known that defects in phospholipid liposomes can be annealed by heating above the gel to liquid-crystal transition temperature of the mixture $(29,30)$, and that dissolution of phosphatidylcholine vesicles by plasma is dependent on temperature (31). In general, an annealing process should allow the formation of more coherent and stable liposomes, leading to comparatively poor adsorption. Previous studies by Notter et al. (14) have shown that CLL can give rapid adsorption when dispersed at higher temperature or energy input than possible with synthetic phospholipid mixtures such as DPPC:PG. This implies that CLL protein either prevents the annealing of defects, or itself creates "fracture sites" in the phospholipid dispersion microstructure. This latter possibility would be consistent with current views of how surfactant apoprotein in natural LS acts to form junctions in the tubular myelin lattice conformation of secreted surfactant in the aqueous phase (3).

The microstructural features shown for $\operatorname{CLL}\left(\mathrm{S}_{\mathrm{i}}\right)$ and $\mathrm{CLL}(\mathrm{V})$ dispersions in Figure 3 do not provide a direct explanation for the different dose dependence these preparations exhibit in restoring pressure-volume mechanics and lung function in surfactant-deficient rat and lamb lungs (21). In these animal models, Notter et al. (21) found that instilled CLL(V) dispersions enhanced pulmonary mechanics and function more effectively than $\operatorname{CLL}\left(\mathrm{S}_{\mathrm{i}}\right)$ dispersions, although the effects of the two became equivalent at high dose $(e . g .100 \mathrm{mg} / \mathrm{kg}$ animal weight in lamb studies). The micrograph of the $\operatorname{CLL}\left(\mathrm{S}_{\mathrm{i}}\right)$ dispersion in Figure $3 A$ does show significant heterogeneity in microstructure at the low levels of sonication energy used $(25 \mathrm{~W}$, see "Materials and Methods"). It is possible that sonication at low levels provides a microstructural sub-population capable of extremely rapid adsorption (very large, thin-walled vesicles), but also leaves large regions of multilamellar aggregates which adsorb quite poorly. However, more sophisticated ultrastructural studies involving measurements of aggregate size distribution and fluidity, and

\footnotetext{
${ }^{4}$ In terms of absolute phospholipid concentration, the $(\pi-t)$ isotherms in Figure 1 demonstrate maximal adsorption for CLL $\left(S_{i}\right.$ and $\left.V\right)$ dispersions, and natural LS suspensions, at a uniform concentration of $0.25 \mathrm{mg}$ phospholipid $/ \mathrm{ml}$. Notter et al. (14) have shown that this behavior extends to even lower bulk phase concentrations in the range $0.06-0.1 \mathrm{mg} / \mathrm{ml}$ for $\mathrm{CLL}\left(\mathrm{S}_{\mathrm{i}}\right)$ dispersions, and for natural $\mathrm{LS}$ with $\mathrm{Ca}^{++}$ ions present.
}

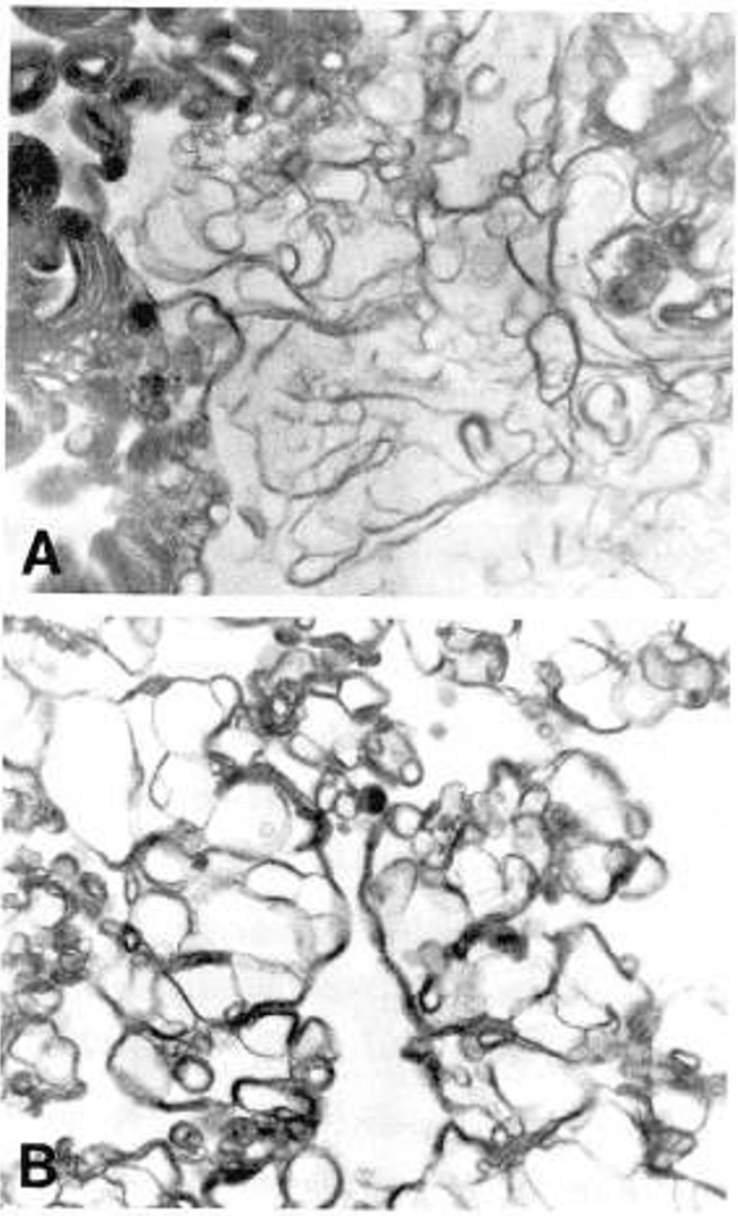

Fig. 3. Electron micrographs of CLL dispersions for conditions in Figure 1A. A, CLL $\left(\mathrm{S}_{\mathrm{i}}\right)$, corresponding to Figure $1 A(\times 17,500) . B, \mathrm{CLL}(\mathrm{V})$, corresponding to Figure $1 A(\times 17,500)$. Tubular myelin, characteristic of optimal adsorption for natural LS, is not present in the microstructure of either CLL(V) or CLL( $\left.\mathrm{S}_{\mathrm{i}}\right)$. See text for details.

perhaps consideration of specific lipid phases (e.g. the Hex II phase), are needed for quantitative correlations between microstructure and adsorption for $\operatorname{CLL}\left(\mathrm{S}_{\mathrm{i}}\right)$ and $\mathrm{CLL}(\mathrm{V})$ dispersions.

Acknowledgment. The assistance of Dr. W. Rosenkrans, Nadia Kutyreff, and Jean Bruns with electron microscopy is gratefully acknowledged.

\section{REFERENCES}

1. Avery ME, Mead J 1959 Surface properties in relation to atelectasis and hyaline membrane disease. Am J Dis Child 97:517-523

2. Notter RH, Shapiro DL 1981 Lung surfactant in an era of replacement therapy. Pediatrics 68:781-789

3. Stratton CJ 1977 The periodicity and architecture of lipid retained and extracted lung surfactant and its origin from multilamellar bodies. Tissue Cell 9:301-316

4. Dobbs LG, Mason RJ, Williams MC, Benson BJ, Sueishi K 1982 Secretion of surfactant by primary cultures of alveolar type II cells. Biochim Biophys Acta 715:118-127

5. Benson BJ, Hawgood S, Williams MC 1984 Role of apoprotein and calcium ions in surfactant function. Exp Lung Res 6:223-236

6. Benson BJ, Williams MC, Sueishi K, Goerke J, Sargeant T 1984. Role of calcium ions in the structure and function of pulmonary surfactant. Biochim Biophys Acta 793:18-27

7. Fujiwara T 1984 Surfactant replacement in neonatal RDS. In: Robertson B, van Golde LMG, Batenburg JJ (eds) Pulmonary Surfactant. Elsevier, Amsterdam, The Netherlands, pp 479-503

8. Notter RH 1984 Surface chemistry of pulmonary surfactant: interactions of individual components. In: Robertson B, van Golde LMG, and Batenburg JJ (eds) Pulmonary Surfactant. Elsevier, The Netherlands, pp 17-65

9. Shapiro DL, Notter, RH, Morin FC, Deluga K, Golub LM, Sinkin R, Weiss 
K, Cox C 1985. A double-blind, randomized trial of a calf lung surfactant extract administered at birth to very premature infants for prevention of the respiratory distress syndrome. Pediatrics 76:593-599

10. Kwong MS, Egan EA, Notter RH, Shapiro DL 1985. A double blind clinical trial of calf lung lipid for the prevention of hyaline membrane disease in extremely premature infants. Pediatrics 76:585-592

11. Enhorning G, Shennan A, Possmayer F, Dunn M, Chen CP, Milligan J 1985. Prevention of neonatal respiratory distress syndrome by tracheal instillation of surfactant: a randomized clinical trial. Pediatrics 76:145-153

12. Lachmann B, and Danzmann E 1984. Adult Respiratory Distress Syndrome. In: Robertson B, Van Golde LMG, Batenburg JJ (eds), Pulmonary Surfactant. Elsevier, The Netherlands pp 505-538

13. Notter RH, Smith S, Taubold RD, Finkelstein JN 1982. Path dependence of adsorption behavior of mixtures containing dipalmitoyl phosphatidylcholine. Pediatr Res 16:515-519

14. Notter, RH, Finkelstein JN, Taubold RD 1983. Comparative adsorption of natural lung surfactant, extracted phospholipids, and artificial phospholipid mixtures to the air-water interface. Chem Phys Lipids 33:67-80

15. Bangham AD, Horne RW 1964 Negative staining of phospholipids and their structural modification by surface-active agents as observed in the electron microscope. J Mol Biol 8:660-668

16. Szoka F, Papahadjopoulos D 1980 Comparative properties and methods of preparation of lipid vesicles (liposomes). Ann Rev Biophys Bioeng 9:467508 .

17. Szoka F, Paphadjopoulos D 1981 Liposomes: preparation and characterization. In: Knight CG (ed) Liposomes: From Physical Structure to Therapeutic Applications. Elsevier, New York, pp 51-82

18. Ostrow MJ (ed) 1984 Liposomes. Marcel Dekker Inc., New York

19. Bermel MA, McBride JT, Notter RH 1984 Lavaged excised rat lungs as a model of surfactant deficiency. Lung 162:99-113

20. Egan EA, Notter RH, Kwong MS, Shapiro DL 1983 Natural and artificial lung surfactant replacement therapy in premature lambs. J Appl Physiol 55:875883

21. Notter RH, Egan EA, Kwong MS, Holm BA, Shapiro DL 1985 Lung surfactant replacement with lipids extracted from bovine lung lavage: effects of dispersion technique, dose, and gestational age. Pediatr Res 19:569-577

22. Metcalfe IL, Pototschnik R, Burgoyne R, Enhorning G 1982 Lung expansion and survival in rabbit neonates treated with surfactant extract. J Appl Physiol 53:838-843

23. Bligh E, Dyer W 1959 A rapid method of total lipid extraction and purification. Can J Biochem 37:911-917

24. Ames B 1966 Assay of inorganic phosphate, total phosphate, and phosphatases. Methods Enzymol 8:115-118

25. Touchstone J, Chen J, Beaver K 1980 Improved separation of phospholipids in thin layer chromatography. Lipids 15:61-62

26. Lowry OH, Rosebrough NJ, Farr AL, Randall RJ 1951 Protein measurement with the folin-phenol reagent. J Biol Chem 193:265-275

27. Kalina M, Pease DC 1977 The preservation of ultrastructure in saturated phosphatidylcholines by tannic acid in model systems and type II pneumocytes. J Cell Biol 74:726-741

28. Obladen M, Popp D, Scholl C, Schwarz H, Jehnig F 1983 Studies on lung surfactant replacement in respiratory distress syndrome: Rapid film formation from binary mixed liposomes. Biochim Biophys Acta 735:215-224.

29. Lawaczeck R, Kainosho M, Chan SI 1976 The formation and annealing of structural defects in lipid bilayer vesicles. Biochim Biophys Acta 443:313330

30. Lawaczeck R, Kainosho M, Girardet J-L, Chan SI 1976. Effects of structural defects in sonicated phospholipid vesicles on fusion and ion permeability. Nature 256:584-586

31. Scherphof G, Morselt H, Regts, J, Wilschut JC 1979. The involvement of the lipid phase transition in the plasma-induced dissolution of multilamellar phosphatidylcholine vesicles. Biochim Biophys Acta 556:196-207. 